INPLASY

PROTOCOL

To cite: Zhao et al. A systematic review and metaanalysis of aortic valve repair for bicuspid versus tricuspid aortic valves. Inplasy protocol 2020120079. doi:

10.37766/inplasy2020.12.0079

Received: 14 December 2020

Published: 14 December 2020

Corresponding author:

Zhong Wu

wuzhong71@scu.edu.cn

Author Affiliation:

Department of

Cardiovascular Surgery, West

China Hospital, Sichuan

University; Chengdu,

Sichuan, P.R. China, 61000

Support: None.

Review Stage at time of this submission: Piloting of the study selection process.

Conflicts of interest:

None.

\section{A systematic review and meta- analysis of aortic valve repair for bicuspid versus tricuspid aortic valves}

Zhao, $\mathrm{X}^{1}$; Sun, K2; Bi, S3; Wu, Z4.

Review question / Objective: In this study, in order to comprehensively analyze the early/late mortality, aortic valverelated reoperation rate and valve regurgitation rate of aortic valve repair for patients with B-AV versus those with T-AV, we plan to carry out this meta-analysis and systematic review.

Condition being studied: Bicuspid aortic valve is the single most common cardiac congenital mal-formation with a prevalence of $1-2 \%$. It is frequently associated with aortic disease including annular ectasia.Since aortic annulus dilatation is often present in patients with incompetent BAV, de Kerchove and colleagues proposed that aortic valve reimplantation is a safer option than traditional aortic valve repair to treat patients with BAV, except in the absence of an aneurysm. These authors recently recorded the findings of aortic valve reimplantation in 440 patients (177 BAV; 76 without aneurysm) and the 10-year reoperation rate was approximately $90 \%$ and comparable for both TAV and BAV [4]. It was also demonstrated by other investigators that BAV has little detrimental impact on the mid-term effects of aortic valve reimplantation.

INPLASY registration number: This protocol was registered with the International Platform of Registered Systematic Review and Meta-Analysis Protocols (INPLASY) on 14 December 2020 and was last updated on 14 December 2020 (registration number INPLASY2020120079).

\section{INTRODUCTION}

Review question / Objective: $\mathbf{n}$ this study, in order to comprehensively analyze the early/ late mortality, aortic valve-related reoperation rate and valve regurgitation rate of aortic valve repair for patients with
B-AV versus those with T-AV, we plan to carry out this meta-analysis and systematic review.

Condition being studied: TBicuspid aortic valve is the single most common cardiac congenital mal-formation with a prevalence 
of $1-2 \%$. It is frequently associated with aortic disease including annular ectasia.Since aortic annulus dilatation is often present in patients with incompetent BAV, de Kerchove and colleagues proposed that aortic valve reimplantation is a safer option than traditional aortic valve repair to treat patients with BAV, except in the absence of an aneurysm. These authors recently recorded the findings of aortic valve reimplantation in 440 patients (177 BAV; 76 without aneurysm) and the 10-year reoperation rate was approximately $90 \%$ and comparable for both TAV and BAV [4]. It was also demonstrated by other investigators that BAV has little detrimental impact on the mid-term effects of aortic valve reimplantation.

\section{METHODS}

Participant or population: The study population is patients undergoing aortic valve repair surgery.

Intervention: Aortic valve repair.

Comparator: Patients with bicuspid aortic valve and tricuspid valve.

Study designs to be included: Case-control study and cohort study.

Eligibility criteria: Studies were included if they satisfied the following criteria: (1) the design was a comparative study of BAV versus TAV; (2) the study population was patients who underwent aortic valve repair. (3) studies that reported outcomes of interest including early or late mortality, reoperation for any reasons; Studies were excluded by the following exclusion criteria: (1) duplicated studies or original data or abstracts without full texts; (2) outcomes of interest were not reported; (3) overlapping of patients among 2 studies with the same primary authors. In this case, the most recent article was included in our meta-analysis.

Information sources: PubMed, Medline and EMBASE databases.
Main outcome(s): Primary outcomes were all-cause overall mortality including early and late mortality. Early mortality was related to 30-day mortality after discharge or in-hospital mortality while late mortality was referred to the death of patients occurred during the follow-up time which contained the 3-year, 5-year and 10-year mortality rate.

Additional outcome(s): Secondary outcomes were aortic valve-related reoperation rate and grade II or more prosthetic valve regurgitation rate. Other outcomes including cerebrovascular accidents and concomitant surgery that reflected the prognosis to some extent. Concomitant surgery concluded coronary artery bypass grafting (CABG), mitral valve repair and tricuspid valve repair.

Quality assessment / Risk of bias analysis: The quality of the individual studies will be evaluated by two authors independently assessed using the Newcastle-Ottawa scale (NOS). The criteria are sample selection (S), comparability (C), and outcome assessment (0). The higher scores represent a better quality of studies. The study will be considered with good quality if it was scored more than 7 points. Publication bias will be measured by the Deeks Funnel Plot Asymmetry Test (considering the presence of $\mathrm{P}<0.05$ publication bias). Stata 14.2 will perform the Deeks Funnel Plot Asymmetry Test (StataCorp, USA).

Strategy of data synthesis: We will evaluate the distribution of potential effect modifiers (publication year, mean age, sample size, male proportion, mean follow-up time, and all preoperative complications) across studies. Then we will summarize the study results used to risk ratios with $95 \%$ confidence intervals (Cls) for dichotomous data and standardized mean differences (SMD) with $95 \%$ Cls for continuous variables. A random-effects model will be chosen over the fixed-effects model to estimate the average treatment effect based on the assumption of differences in the treatment effect and/or sampling variability between studies. This 
assumption will be tested with Cochran's Q-test ( $p$-value < 0.1), 12 statistic and $\tau 2$ statistic for heterogeneity expressed as a percentage.

Subgroup analysis: None.

Sensibility analysis: We will use the influence analyses to detect studies that influence the overall estimate of our metaanalysis the most and show relative plots as measured by 12 ordered by heterogeneity (low to high). Also, we will select funnel-plot-based methods to resolve publication bias, which can affect the validity and generalization of conclusions in meta-analysis.

Country(ies) involved: China.

Keywords: Aortic valve repair; bicuspid aortic valve; tricuspid aortic valve; metaanalysis.

Contributions of each author:

Author 1 - Xueshan Zhao.

Author 2 - Kaibo Sun.

Author 3 - Siwei Bi.

Author 4 - Zhong Wu. 